\title{
Air-damping effects on developing velocity profiles in flowing soap films
}

\author{
M.-J. Huang ${ }^{\text {a) }}$ \\ Department of Mechanical Engineering, National Taiwan University, Taipei 106, Taiwan \\ C.-Y. Wen \\ Department of Mechanical Engineering, Da-Yeh University, Chang-Hwa 51505, Taiwan \\ I.-C. Lee \\ Department of Mechanical Engineering, National Taiwan University, Taipei 106, Taiwan \\ C.-H. Tsai \\ Department of Mechanical Engineering, Da-Yeh University, Chang-Hwa 51505, Taiwan
}

(Received 28 January 2004; accepted 10 August 2004; published online 5 October 2004)

\begin{abstract}
The continuously running gravity-driven soap-film tunnel is a device suitable for the study of two-dimensional flows. In this innovative device, the films start from a reservoir, run over a vertical wire frame and get pulled by the gravity force. Despite its simple design and successful applications in two-dimensional flows, its working mechanisms are not fully understood. In the present work, the laminar velocity profiles of freely suspended flowing soap films are examined theoretically and experimentally. A complete momentum integral analysis is performed including boundary layers developed within the channel on the film as well as beside the film in the air. The theoretical results are compared with the experimental measurements via laser Doppler velocimetry. Reasonable agreements are observed. Although the gravity force speeds up the film motion, the acceleration is significantly slowed down, but not completely, by the air friction. The growth of the boundary layers developed on the film is also damped by the air friction so that across the film channel the velocity profile is mostly uniform. Moreover, a saturated boundary layer thickness seemingly exists when the thinning due to acceleration and the thickening due to viscous diffusion are in balance. (C) 2004 American Institute of Physics. [DOI: 10.1063/1.1802131]
\end{abstract}

\section{INTRODUCTION}

A common characteristic of geostrophic atmospheric or oceanic flows is their large surface area-to-depth ratio, which makes them exhibit apparent two-dimensional (2D) turbulent behavior. Since the publication of the seminal works of Kraichnan $^{1}$ and Batchelor, ${ }^{2}$ 2D turbulence has been given much more attention in numerical and theoretical investigations than in experimental ones. The main problem in simulating 2D flows in the laboratory is due to the difficulty of isolating 2D flows from evolving implicit three-dimensional (3D) instabilities.

In the past few decades, soap films were nominated to produce and study 2D hydrodynamics. ${ }^{3-5}$ Couder $^{3}$ stretched $^{-1}$ the film on a large frame and used it as a two-dimensional towing tank. Gharib and Derango ${ }^{4}$ designed and built the first continuously running horizontal soap-film tunnel. In this tunnel, a suspended horizontal soap film is set in motion in a horizontal long frame using a planar water jet as a pulling mechanism. Kellay et al. ${ }^{5}$ recently proposed and tested another continuously running vertical soap-film tunnel. In their setup, a soap film is bounded at its edges by two parallel vertical nylon fishing wires and the flow is driven by the force of gravity. These films, which have a thickness ranging from 0.1 to $10 \mu \mathrm{m}$, are $2 \mathrm{D}$ to an excellent approximation. The ratio of the size of coherent wake vortex structures to the

\footnotetext{
${ }^{a)}$ Author to whom correspondence should be addressed. Telephone: +8862 23673984. Fax: +8862 23631755. Electronic mail: mjhuang@ntu.edu.tw
}

film thickness routinely exceeds $10^{4}$. A variety of $2 \mathrm{D}$ experiments were then performed in these soap films and have shown certain features of turbulent flow that resemble those anticipated for a true 2D system (e.g., Couder and his co-workers, ${ }^{3,6,7}$ Gharib and Derango, ${ }^{4}$ Beizaie and Gharib, ${ }^{8}$ Kellay et al., ${ }^{5} \mathrm{Wu}$ et al., ${ }^{9}$ Rutgers et al., ${ }^{10}$ Martin et al., ${ }^{11}$ Rivera et al., ${ }^{12}$ and Wen and Lin $^{13}$ ).

Despite the simple design and successful applications in two-dimensional flows of soap-film tunnels, their detailed working mechanisms are not fully understood. Only a few papers conducted the theoretical analyses to help understand their working mechanisms. Chomaz ${ }^{14}$ presented a complete analysis of the three-dimensional fluid dynamics in a horizontal soap film, using the asymptotic lubrication theory, which only assumes that the thickness of the film is small compared to the characteristic length scale of the in-plane flow. In this paper, the author did proper demonstrations to validate whether soap films obey the classical twodimensional Navier-Stokes equations. The leading-order approximation for the dynamics of a flat soap film was derived, which gives both the physics of the equilibrium at play in the free film and the order of magnitude of the neglected effects. The evolution equations governing the leading-order film thickness, two-dimensional velocities (locally averaged across the film thickness), average surfactant concentration in the interstitial liquid, and the surface concentration are given and compared to similar results from literature. This master model takes into account a large number of physical 
effects: film elasticity, film stiffness (curvature effect), viscosity, diffusion, arbitrary large variations of thickness, adsorption and desorption of the soap (solubility of the soap), and nonuniform initial soap concentration. A sufficient condition for the film velocity distribution to comply with the two-dimensional Navier-Stokes equations is shown to be that the typical flow velocity should be small compared to that Marangoni elastic wave velocity, i.e., small Mach number. In that case, the thickness variations are slaved to the velocity field in a very specific way that seems consistent with recent experimental observations in the near wake. Beizaie and Gharib $^{8}$ derived a force balance equation between the shear force produced by the water sheet and the opposing pulling forces of reservoir and boundary layer frictions in the horizontal soap-film tunnel. The thermodynamic equilibrium relationship between two external monolayers of surfactants and a slab of surfactant solution in between was modeled by Langmuir's adsorption theory. When a film is drawn from the reservoir to the water sheet, the surfactant molecules start migrating in the same direction. The dragged film pulls more surfactant to restore the thermodynamic equilibrium, due to Marangoni elasticity, and thus a flow is established. The horizontal film flow soon reaches an equilibrium rate as required by the force balance mentioned above. The results also show that both film velocities and film flow rates increase to a saturation level with water sheet velocity, and the average film thickness depends on the surfactant concentration. The works of both Chomaz ${ }^{14}$ and Beizaie and Gharib ${ }^{8}$ exclude the air damping effects.

The air/liquid interfaces on either side of the film are actually subject to nonlinear shear by the surrounding air (and its boundary layer). Couder $e t a l .{ }^{6}$ fully appreciated the importance of air damping and made an estimate of the effect of air on the damping of turbulent vortices in their pioneering work on the hydrodynamics of soap films. Rutgers et $a l .{ }^{10}$ have examined experimentally and theoretically the laminar velocity profiles of vertically flowing soap films. Their measurements concentrated on the transverse film velocity profiles and their theoretical goal was limited to explaining the measured transverse velocity profiles at large streamwise distances from the injection point of the soap solution, by making the assumption that the transverse velocity profiles are independent of the streamwise distance, i.e., soap films have reached a terminal velocity. It is found that air surrounding a vertical film plays a primary role. The laminar velocity profiles in the vertical soap-film tunnel are governed by an interplay of boundary layers in air and in the film itself. Air then compromises the two dimensionality of the film since the air boundary layers extend over 1000 times the film thickness into the third $(z)$ dimensions. Since the air motion is induced by the flowing soap film, the film is modeled as a semi-infinite plate whose leading edge moves with a velocity through stationary air. The air drag on the film was formulated using Prandtl laminar boundary layer approximation and general features of the transverse velocity profiles were successfully described. However, near the edges of the channel, where Prandtl laminar boundary layer theory breaks down because the leading edge cannot be well defined for the channel walls, artificial boundaries were introduced into the vertical soap-film tunnel in the form of a thin wire and a thin plastic sheet. Three theoretical models, taking into account the interactions between film and air and between film and solid boundaries, were then introduced to explain the measured transverse velocity profiles accordingly. The first model is referred to as the one-dimensional model. The stress due to the air is linearly approximated by dividing the film velocity by the thickness of the air boundary layer. The solution leaves three free fitting parameters and requires the unphysical condition that the velocity does not go to zero at the wire. The second model, referred to as the twodimensional wire model, calculated the velocity field in the air around the film, in addition to the velocity profile in the soap film itself. The calculation is a variant of laminar flow through a rectangular pipe. Flows in the air and film are driven by the force of gravity on the film instead of the pressure gradient. The third model, referred to as the twodimensional sheet model, is the same as the second model except for the wire that is replaced by a sheet, which is used to separate the rectangular pipe into two equal parts. However, none of these models reflects the normal operating conditions of the vertical soap-film tunnel.

In the present work, we further study the working mechanisms of a vertical soap-film tunnel. The theoretical and experimental efforts are focused on understanding the air-damping influences on developing velocity profiles in flowing soap films under normal experimental conditions as well as under no-slipping boundary conditions. The paper is organized as follows. Section II describes the experimental apparatus and general characteristics of the flow. In Sec. III, a momentum integral analysis is performed including boundary layers developed within the channel on the film and those beside the film in the air. In Sec. IV, the theoretical results of the transverse velocity profiles and the development of the centerline velocity in the streamwise direction are compared with the experimental measurements.

\section{THE EXPERIMENTS}

The experiments were conducted in a vertical soap-film tunnel. As shown in Fig. 1, the tunnel consists of an upper supplying reservoir and a lower collecting reservoir connected by two nylon fishing wires. The wires expand from the injection point over the first $85 \mathrm{~cm}$ section, and are parallel after that. The soap film is then bounded at its edge by these two thin wires, with the parallel vertical portion as the test section. The tunnel height is about $2.5 \mathrm{~m}$; the width (typically $3-10 \mathrm{~cm}$ ) is adjustable by varying the separation between the nylon wires. The flow is driven by gravity. The film velocities, ranging between 0.8 and $2.0 \mathrm{~m} / \mathrm{s}$, can be instantly tuned by changing the injection rate of the valve. In this study, the tunnel width was fixed at $3 \mathrm{~cm}$ and the film velocity was kept below $2 \mathrm{~m} / \mathrm{s}$ to avoid the film surface instability out of the plane defined by the guide wires. The tunnel is shielded from the room by curtains to prevent air currents from disturbing the film. The tunnel is also placed on weak-spring passive isolation systems to minimize the effects of floor vibrations. All the experiments were conducted at night when all disturbances were minimal. Since 


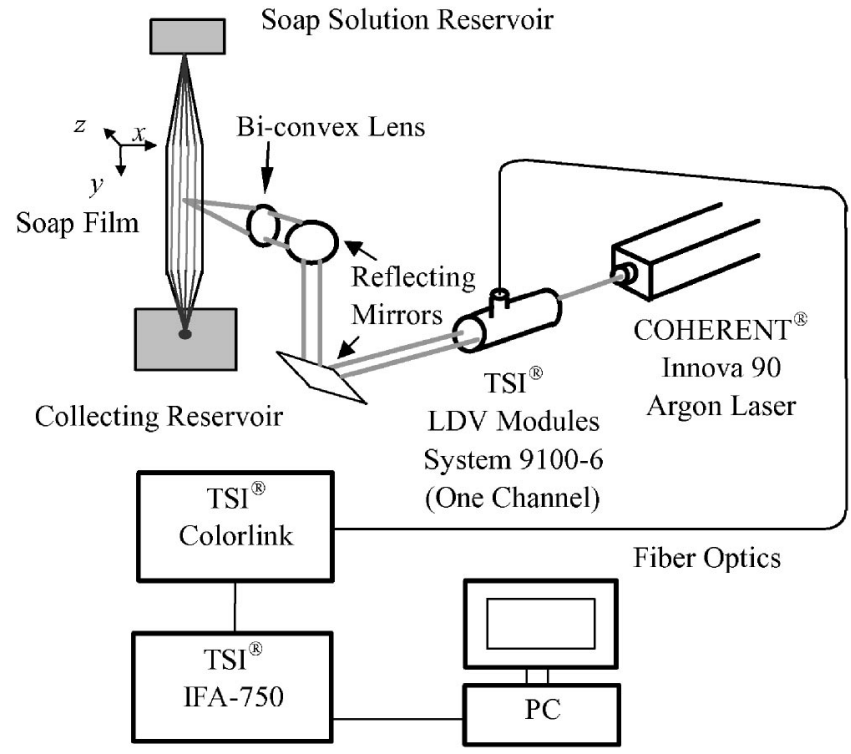

FIG. 1. Schematics of vertical soap-film tunnel and laser Doppler setup for velocity measurement.

the film is continuously fed, evaporation does not pose a problem for either tunnel.

Experiments were performed with mixtures of distilled water and $0.5 \%, 1.0 \%$, and $1.5 \%$ commercial liquid detergent (Ivory Dishwashing Liquid) by weight, respectively. No glycerol was added to the mixture. Low concentrations of soap solutions ensure the Newtonian behavior of the soap films. In order to estimate the film viscosity, the approach of Gharib and Derango ${ }^{4}$ was adopted. Gharib and Derango ${ }^{4}$ have shown that the two-dimensional vortex shedding process of a circular cylinder at a macroscopic level has a strong similarity to its three-dimensional counterpart for Reynolds number (based on the diameter of the cylinder) up to about 180. The vortex shedding process can then be used as a practical method to estimate film viscosity. Roshko's famous equation ${ }^{15}$ shows that the Strouhal number $\mathrm{St}\left(\equiv f d / V_{f}\right)$ and the Reynolds number $\operatorname{Re}\left(\equiv V_{f} d / \nu\right)$ are related by $\mathrm{St}=0.212$ $-4.5 / \mathrm{Re}$. Here, $f$ is the vortex shedding frequency, $V_{f}$ is the free-stream film velocity, $d$ is the cylinder diameter, and $\nu$ is the kinematic viscosity. By measuring the velocity and shedding frequency of a known size cylinder in the film and then using St and Roshko's equation to obtain Re, the film viscosity is determined consequently. The film viscosities for $0.5 \%$, $1.0 \%$, and $1.5 \%$ soap-film solutions were estimated to be $3.74 \times 10^{-5} \mathrm{~m}^{2} / \mathrm{s}, 4.51 \times 10^{-5} \mathrm{~m}^{2} / \mathrm{s}$, and $4.9 \times 10^{-5} \mathrm{~m}^{2} / \mathrm{s}$, respectively.

A commercial one-component laser Doppler velocimetry system (TSI IFA-750 assembly), powered by a $5 \mathrm{~W}$ Argon laser of wavelength $514.5 \mathrm{~nm}$, was used to measure velocity profiles. One biconvex lens and two reflecting mirrors were carefully aligned and mounted on a translation stage that allowed the scattering volume to be scanned on the soap film in the streamwise and transverse directions. The soap solution was seeded with $0.6 \mu \mathrm{m}$ diameter polystyrene spheres. For the current vertical soap-film tunnel, the longitudinal free-stream turbulence intensity does not exceed $1 \%$.

Also shown in Fig. 1 is the coordinate system used for

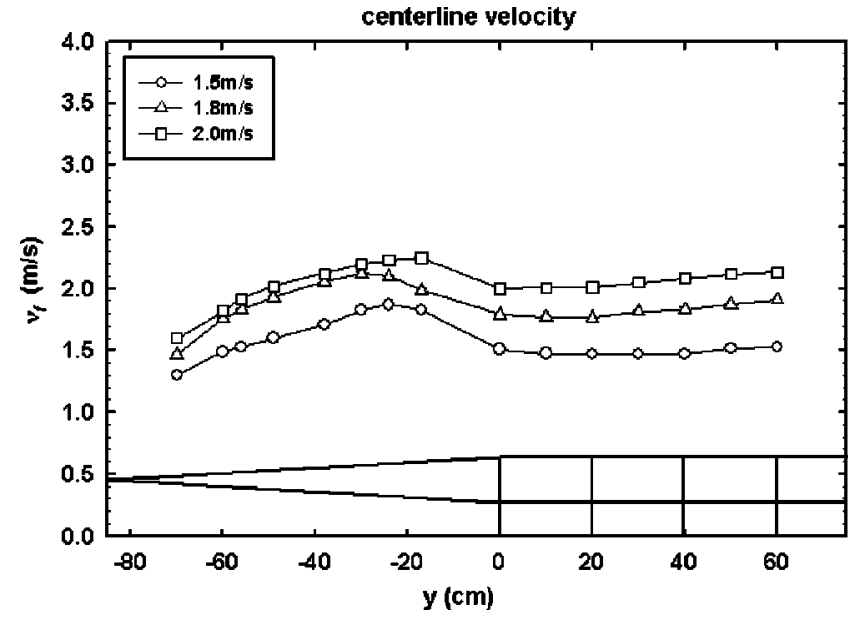

FIG. 2. Velocities as measured at center of vertical soap-film tunnel along $y$ direction from injection point. Three different injection rates were used. For a low injection rate $(0), V_{f}=1.5 \mathrm{~m} / \mathrm{s}$ at $y=0$, whereas for the other two higher injection rates $(\triangle, \square), V_{f}=1.8$ and $2.0 \mathrm{~m} / \mathrm{s}$ at $y=0$, respectively.

the current study, where the flow is in the vertical $y$ direction, $x$ axis is transverse to the flow, and $z$ axis is pointing toward the air. The origin of the coordinate system is located at the point on the film centerline where the channel stops diverging.

Figure 2 shows the streamwise development ( $y$ dependence) of the centerline velocity $V_{f}$ for different injection rates. As the film emerges from the injection point at the top of the tunnel, it moves with the free-falling velocity and accelerates for a short distance. The film is then slowed by air friction on its two surfaces. A larger injection rate results in a thicker film and hence a higher $V_{f}$. The leveling of every $V_{f}$ with increasing $y$ in the parallel test section might suggest that terminal velocities are being approached. For a low injection rate with $V_{f}=1.5 \mathrm{~m} / \mathrm{s}$ at $y=0, \partial V_{f} / \partial y \fallingdotseq 0.15 \mathrm{~s}^{-1}$, whereas for the other two higher injection rates with $V_{f}$ $=1.8$ and $2.0 \mathrm{~m} / \mathrm{s}$ at $y=0, \partial V_{f} / \partial y \fallingdotseq 0.34 \mathrm{~s}^{-1}$.

Figure 3 presents typical transverse film velocity profiles $v_{f}(x)$ measured at four sections $y=0,20,40$, and $60 \mathrm{~cm}$, for $V_{f}=1.8 \mathrm{~m} / \mathrm{s}$ at $y=0$. The velocity profiles evolve and re-

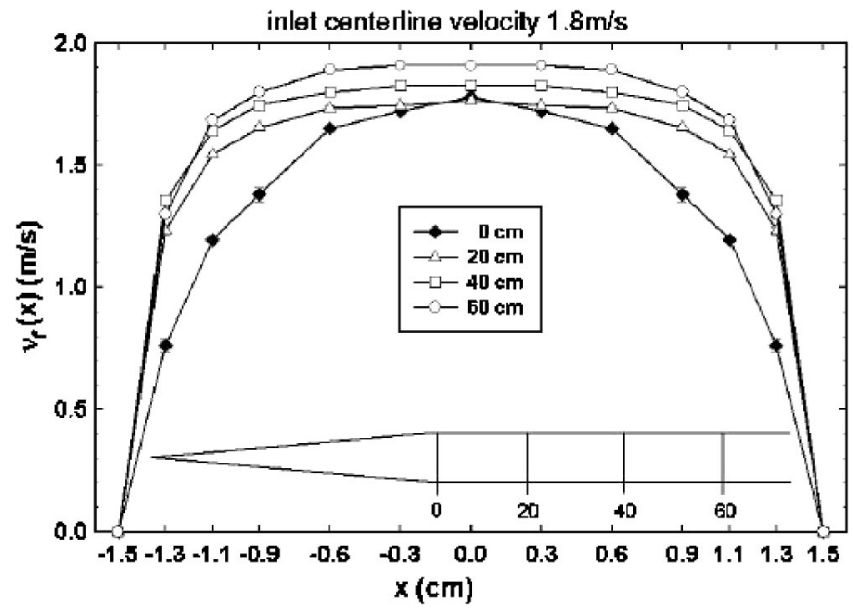

FIG. 3. Typical transverse film velocity profiles $v_{f}(x)$ measured at four sections in the interval $y=0,20,40$, and $60 \mathrm{~cm}$, for $V_{f}=1.8 \mathrm{~m} / \mathrm{s}$. 


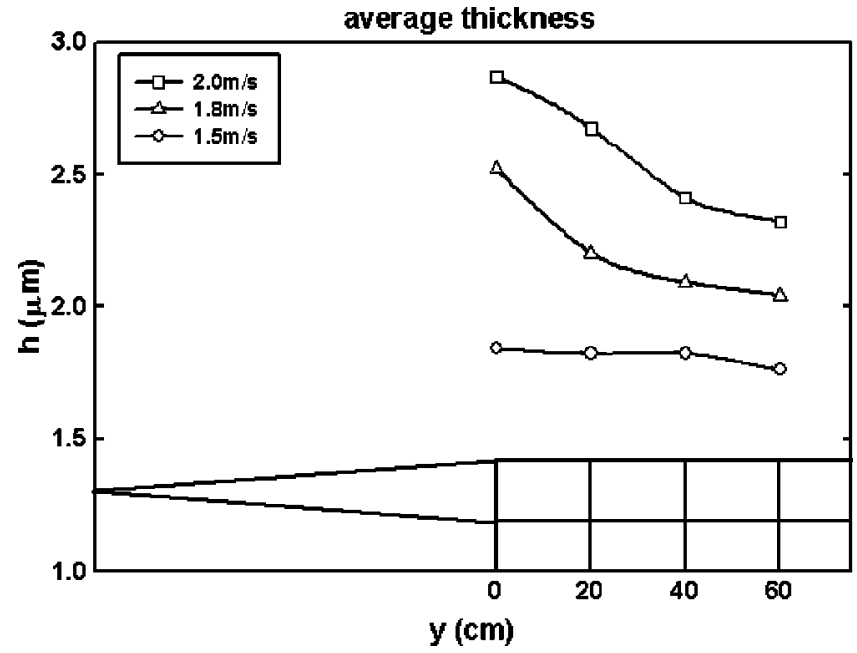

FIG. 4. Variation of average film thickness $h$ along $y$ axis for different injection rates.

semble that of plug flows. The slightly different velocity profile at $y=0 \mathrm{~cm}$ is due to the transition from the diverging section to the parallel section. The film thickness $h$ along $y$ axis can be deduced from the measured volume flow rate and velocity profiles. The volume flow rate was determined by measuring the volume of depletion in the upper soap solution reservoir in a certain period of time. From visual observation, the film shows uniform color over the channel width, except within 1-3 $\mathrm{mm}$ of the bounding wires where a few color fringes are apparent, indicating a slightly larger thickness variation. Therefore, $h$ is assumed constant throughout the entire width of the channel and equals the ratio of the volume flow rate and the integral of the velocity profile $v_{f}(x)$ over the channel width. The variation of $h$ along $y$ axis is shown in Fig. 4, for different injection rates. Higher injection rates yield thicker films. The average film thickness $h$ decreases gradually as the film flows downstream. The total vertical variation of $h$ ranges from $4 \%$ to $18 \%$ in the interval $0<y<60 \mathrm{~cm}$, depending on the fluid injection rate, and the corresponding $\partial h / \partial y$ ranges from $-1.3 \times 10^{-7}$ to -9.2 $\times 10^{-7}$. All the above-mentioned observations are similar to those of Rutgers et al. ${ }^{10}$

Because of the small surface curvatures consequent to the small variations of thickness, the Laplace pressure (curvature effect) is neglected in the later theoretical models. Rusanov and Krotov ${ }^{16}$ have shown that the presence of impurities or different surfactants in commercial soap solutions makes the equilibrium between the surface concentration $\Gamma$ and the bulk concentration $c$ more difficult to reach and an adsorption-desorption time up to an order of $1 \mathrm{~s}$ or more can be observed. The traveling time for a film element is estimated to be less than $0.4 \mathrm{~s}$ in the interval $0<y<60 \mathrm{~cm}$. On the basis of the above arguments, the surface tension gradient due to the adsorption and desorption effect is also neglected in the following mathematical models.

\section{MATHEMATICAL MODELS}

To investigate the effect of air drag on the film motion, instead of pursuing a full numerical solution of the steady

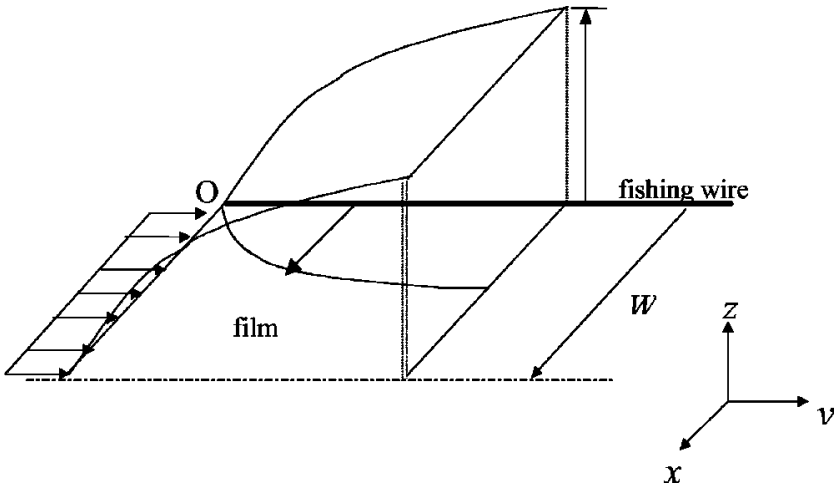

FIG. 5. Schematic representation of a segment of soap-film volume for momentum integral analysis.

three-dimensional, multiphase, Navier-Stokes equations, a simpler integral analysis is performed as follows to capture some fundamental physics.

\section{A. Velocity profiles}

As shown in Fig. 5, the film channel width is $2 W$ and boundary layers develop within the channel on the film as well as in the air on both sides of the film. The inlet is defined at $y=0$. For simplicity, several assumptions are made. First, the film velocity outside the film boundary layer (the film potential region), $V_{f}$, is assumed to be a function of the downstream distance $y$ only. Second, the film velocity profile $v_{f}$ within the boundary layer is approximated as a polynomial of degree 2 satisfying zero velocities on the fishing wires, that is,

$$
\frac{v_{f}}{V_{f}}=2 \frac{x}{\delta_{f}}-\left(\frac{x}{\delta_{f}}\right)^{2}
$$

for $0 \leqslant x \leqslant \delta_{f}$, where $\delta_{f}=\delta_{f}(y)$ is the film boundary layer thickness. The velocity profile for air neighboring the film boundary layer regions will be denoted as $v_{a}$, and that for air next to the film potential region will be $V_{a}$. Because the air is driven by the film motion, in use of a polynomial profile of degree 2, the air velocity $V_{a}$ is approximated as

$$
\frac{V_{a}}{V_{f}}=\left(1-\frac{z}{\delta_{a}}\right)^{2}
$$

where $\delta_{a}=\delta_{a}(y)$ is the air boundary layer thickness. Consequently, $V_{a}=V_{a}(y, z)$. The air velocity $v_{a}$ neighboring the film boundary layer region is much more complicated because $v_{f}$ is a function of both $x$ and $y$. In the present model, for simplicity, the air velocity will be assumed to be proportional to its driving velocity. Thus, the air velocity $v_{a}$, if concerned, will be assumed to be

$$
\frac{v_{a}(x, y, z)}{v_{f}(x, y)}=\frac{V_{a}(y, z)}{V_{f}(y)} .
$$

In other words, using (1) and (2),

$$
\frac{v_{a}}{V_{f}}=\left(1-\frac{z}{\delta_{a}}\right)^{2}\left[2 \frac{x}{\delta_{f}}-\left(\frac{x}{\delta_{f}}\right)^{2}\right] .
$$




\section{B. Integral analysis of mass and momentum conservations}

The mass and momentum conservations of the above four regions are sought now. As mentioned in Sec. II, the film thickness $h$ is assumed to be uniform across the film channel. The stretching of the film due to the surface tension is also neglected. With all the above assumptions, the conservations of mass and momentum for the two interested regions on the film, namely, the film potential region (a control volume of $h d y \int_{\delta_{f}}^{W} d x$ ) and the film boundary layer region (a control volume of $h d y \int_{0}^{\delta_{f}} d x$ ), require

$$
\begin{aligned}
& \rho_{f} h V_{f} \frac{d V_{f}}{d y}=\rho_{f} h g-h \frac{d p}{d y}-2 \tau_{a V}, \\
& \rho_{f} h \frac{d}{d y} \int_{0}^{\delta_{f}} v_{f}^{2} d x-\rho_{f} h V_{f} \frac{d}{d y} \int_{0}^{\delta_{f}} v_{f} d x \\
& =\rho_{f} h g-h \frac{d p}{d y}-2 \int_{0}^{\delta_{f}} \tau_{a v} d x-\tau_{f} h .
\end{aligned}
$$

Terms on the left-hand sides of Eqs. (5) and (6) are the net flow rates of $y$ momentum out of the two interested control volumes. Terms on the right-hand sides are the gravity force, pressure force, air drag, and film friction, respectively. The film friction term in Eq. (5) is absent because the film potential region is being discussed. On the other hand, the conservations of mass and momentum in the air region neighboring the film potential region (a control volume of $d y \int_{0}^{\delta_{a}} \int_{\delta_{f}}^{W} d x d z$ ) result in

$$
\rho_{a} \frac{d}{d y}\left\{\left(W-\delta_{f}\right) \int_{0}^{\delta_{a}} V_{a}^{2} d z\right\}=\left(W-\delta_{f}\right) \tau_{a V},
$$

in which the air gravity force and the air pressure gradient are ignored.

The air drag terms and the film friction term in Eqs. (5) and (6) are attainable by assuming Newtonian fluids for both the soap solution and the air, as well as by using the velocity profiles discussed in the preceding section. Four unknown variables $V_{f}(y), \delta_{f}(y), \delta_{a}(y)$, and $h(y)$ are there nonetheless. The fourth equation required for a closure of the problem comes from the total mass conservation of the soap solution in the channel, which is

$$
Q_{f}=\int_{0}^{\delta_{f}} v_{f} h d x+V_{f} h\left(W-\delta_{f}\right),
$$

where $Q_{f}$ is half the soap solution volume flow rate.

It is worth mentioning here that the film surface tension is not considered in the present models. The main purpose of the present investigation is to understand if there is a possibility of obtaining a terminal film velocity. When the film motion is accelerated due to gravity, its thickness is generally decreasing in the downstream direction, which means the surface tension is pulling the film downward as well. An inclusion of the film surface tension therefore decreases the possibility. On the other hand, if there is a terminal velocity, the film thickness should be unchanged (for a constant volume flow rate) and therefore there is negligible net film surface tension force.

\section{Nondimensionalization}

After the shear stresses have been computed by using the assumed velocity profiles and assuming Newtonian fluids, the resulting equations can be further rearranged as follows:

$$
\begin{aligned}
\delta_{f}^{*}= & 3\left(V_{f}^{*} h^{*}-1\right) / V_{f}^{*} h^{*}, \\
\frac{d V_{f}^{*}}{d y^{*}}= & \frac{1}{F r^{2}} \frac{1}{V_{f}^{*}}-\frac{4}{\operatorname{Re}_{a}} \frac{\rho_{a}}{\rho_{f}} \frac{W}{h_{0}} \frac{1}{\delta_{a}^{*} h^{*}}, \\
\frac{d h^{*}}{d y^{*}}= & \frac{1}{2 \mathrm{Fr}^{2}} \frac{\left(7-9 V_{f}^{*} h^{*}\right)}{V_{f}^{* 3}}+\frac{4}{\operatorname{Re}_{a}} \frac{\rho_{a}}{\rho_{f}} \frac{W}{h_{0}} \frac{\left(4-3 V_{f}^{*} h^{*}\right)}{\delta_{f}^{*} h^{*} V_{f}^{* 2}} \\
& +\frac{5}{3 \operatorname{Re}_{f}} \frac{h^{* 2}}{\left(V_{f}^{*} h^{*}-1\right)},
\end{aligned}
$$

$$
\frac{d}{d y^{*}}\left[V_{f}^{* 2}\left(1-\delta_{f}^{*}\right) \delta_{a}^{*}\right]=\frac{10}{\operatorname{Re}_{a}} \cdot \frac{V_{f}^{*}\left(1-\delta_{f}^{*}\right)}{\delta_{a}^{*}},
$$

where variables (starred) have been nondimensionalized using the average inlet film velocity $\left(V_{0}\right)$, half the channel width $(W)$, and the inlet film thickness $\left(h_{0}\right)$; that is, $y^{*}$ $=y / W, h^{*}=h / h_{0}, V_{f}^{*}=V_{f} / V_{0}, \delta_{f}^{*}=\delta_{f} / W$, and $\delta_{a}^{*}=\delta_{a} / W$. The soap solution volume flow rate is thus $2 Q_{f}=2 h_{0} V_{0} W$. The dimensionless parameters are defined as $\operatorname{Re}_{a} \equiv \rho_{a} V_{0} W / \mu_{a}$, $\operatorname{Re}_{f} \equiv \rho_{f} V_{0} W / \mu_{f}$, and $\mathrm{Fr} \equiv V_{0} / \sqrt{g W}$, respectively. Terms involving the Froude number Fr come from the gravity effect. Those involving $\mathrm{Re}_{a}$ in Eqs. (10)-(12) are the mutual interactions between the air and the film motions. The last term in Eq. (11) regarding $\operatorname{Re}_{f}$ comes from the film friction.

Notice that the air drag effect $\left(\tau_{a v}\right)$ on the film boundary layer regions has been ignored in Eq. (11). Therefore, the air drag influences the film boundary layer motion only indirectly through its effect on $V_{f}$. If, instead, Eqs. (3) or (4) is employed to evaluate $\tau_{a v}$, Eq. (11) should be modified to become

$$
\begin{aligned}
\frac{d h^{*}}{d y^{*}}= & \frac{1}{2 \operatorname{Fr}^{2}} \frac{\left(7-9 V_{f}^{*} h^{*}\right)}{V_{f}^{* 3}}+\frac{4}{\operatorname{Re}_{a}} \frac{\rho_{a}}{\rho_{f}} \frac{W}{h_{0}} \frac{\left(2 V_{f}^{*} h^{*}-1\right)}{\delta_{f}^{*} h^{*} V_{f}^{* 2}} \\
& +\frac{5}{3 \operatorname{Re}_{f}} \frac{h^{* 2}}{\left(V_{f}^{*} h^{*}-1\right)} .
\end{aligned}
$$

The model in use for Eq. (11) will then be denoted as model (a) and that in use for Eq. (13) as model (b) in the following discussions.

The above equations are then numerically solved by employing a fourth order Runge-Kutta method. The initial conditions $V_{0}$ and $h_{0}$ used are extracted from the experimental data. The initial value of film boundary layer thickness $\delta_{f}(y=0)$ is computed from Eq. (9). The initial value of air boundary layer thickness $\delta_{a}(y=0) \equiv \delta_{a}^{\text {inlet }}$ turns out to be the only free parameter in the present mathematical model. 
TABLE I. Flow conditions of three cases: $2 W=3 \mathrm{~cm}, \rho_{f}=998.2 \mathrm{~kg} / \mathrm{m}^{3}, \rho_{a}$ $=1.205 \mathrm{~kg} / \mathrm{m}^{3}, \nu_{a}=1.51 \times 10^{-5} \mathrm{~m}^{2} / \mathrm{s}$, and $g=9.8 \mathrm{~m} / \mathrm{s}^{2}$.

\begin{tabular}{lccc}
\hline \hline & Case 1 & Case 2 & Case 3 \\
\hline$\nu\left(\mathrm{m}^{2} / \mathrm{s}\right)$ & $3.74 \times 10^{-5}$ & $4.51 \times 10^{-5}$ & $4.90 \times 10^{-5}$ \\
$V_{0}(\mathrm{~m} / \mathrm{s})$ & 1.297 & 1.353 & 1.55 \\
$h_{0}(\mu \mathrm{m})$ & 1.84 & 2.52 & 2.87 \\
$V_{\text {inlet }}(\mathrm{m} / \mathrm{s})$ & 1.50 & 1.80 & 2.00 \\
$\delta_{a}^{\text {inlet }}(\mathrm{cm})$ for model $(\mathrm{a})$ & 0.310 & 0.960 & 0.805 \\
$\delta_{a}^{\text {inlet }}(\mathrm{cm})$ for model $(\mathrm{b})$ & 0.198 & 0.540 & 0.439 \\
\hline \hline
\end{tabular}

\section{COMPARISONS AND DISCUSSIONS}

To compare the model results with the experimental measurements, the measured data at position $y=0$ where the channel stops diverging are taken as the inlet conditions for the models and are listed in Table I. The inlet air boundary layer thickness $\delta_{a}^{\text {inlet }}$ is so adjusted to fit the measured centerline velocities with the least root-mean-square error.

The first variable to be compared is the dimensional centerline velocities, $V_{f}$, in Fig. 6. The results without consideration for the air drag that nullifies the corresponding terms in the governing equations are also plotted for a comparison. As seen, the accelerations of the film motion in all cases are significantly reduced by the air drag. As $y$ increases, the computed $V_{f}$ increases initially and then decreases for a short distance before it increases monotonically again, when the volume flow rates are higher (case 2 and case 3 ). The increase is attributed to the gravity and the decrease is caused by the air drag and the film friction. The air drag is also responsible for the big dip observed in the curve of the computed $V_{f}$ when the volume flow rate is low (case 1), in which a small $\delta_{a}^{\text {inlet }}$ was used (thus close to the leading-edge singularity). It is probably not physically real. An examination of the computed $V_{f}$ at very large downstream distances shows slowly increasing $V_{f}$, implying no so-called terminal velocity

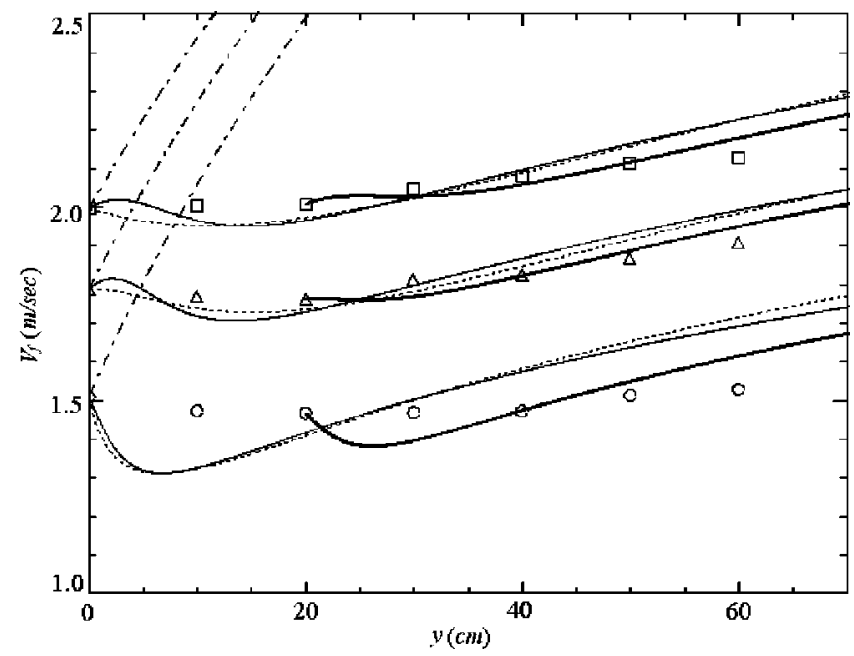

FIG. 6. The streamwise development of the computed centerline film velocity compared with experimental data (symbols). Solid, dotted, and dash dotted lines are the results of model (a), model (b), and no-air-drag model, respectively. Thick solid curves are the results of using flow data at $y$ $=20 \mathrm{~cm}$ as initial conditions from model (a).

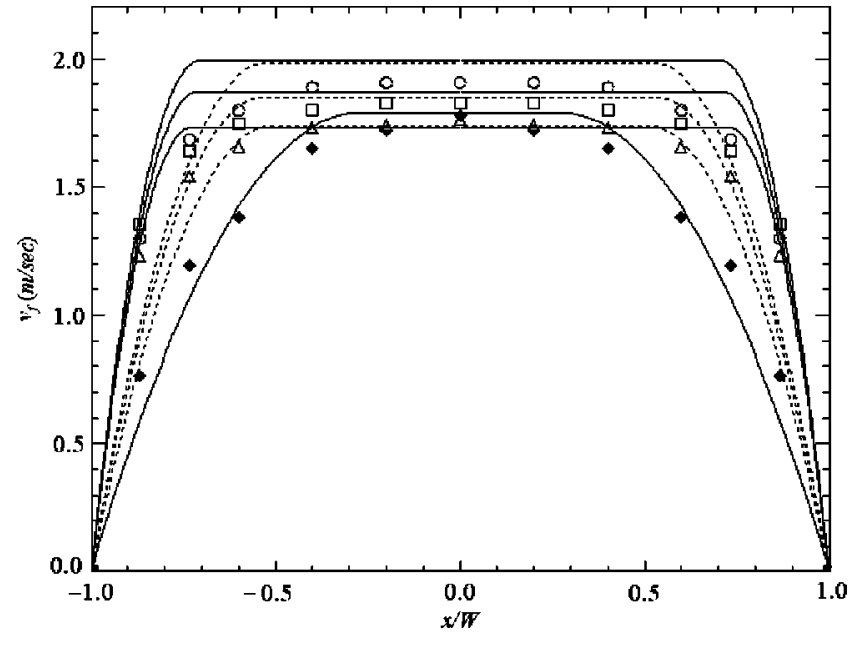

FIG. 7. The computed velocity profiles for case 2 at several downstream distances. Solid lines and dotted lines are the results of models (a) and (b). Symbols are experimental measurements: at $y=0 \mathrm{~cm}$ (inlet); $\Delta$ at $y$ $=20 \mathrm{~cm}$; at $y=40 \mathrm{~cm} ; \bigcirc$ at $y=60 \mathrm{~cm}$.

or no fully developed velocity profile. This is not out of expectation, because there is no way in the present layout to prevent the air boundary layers from growing, and the film friction is not strong enough to balance gravity. This prediction is different from that of Rutger et al., ${ }^{10}$ whose wire and sheet models confine the growth of the air boundary layers within a rectangular channel. Finally, superimposed also in Fig. 6 are the centerline velocities predicted by taking the flow data at $y=20 \mathrm{~cm}$ as the initial conditions for the model (a). Similar trends are observed but agreement is improved. Because the trends are similar, in the followings, only results by using initial conditions at $y=0 \mathrm{~cm}$ are discussed.

The computed velocity profiles at several downstream distances are compared with the experimental measurements in Figs. 7 and 8. Considering the air motion neighboring the film boundary layer regions, model (b) gives a thicker film boundary layer thickness which is closer to the experimental

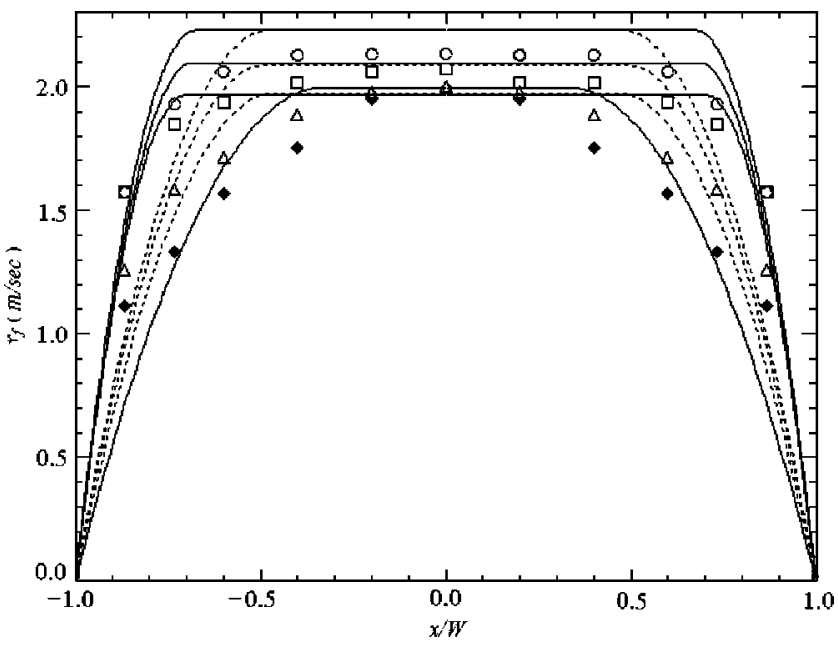

FIG. 8. The computed velocity profiles for case 3 at several downstream distances. Solid lines and dotted lines are the results of models (a) and (b). Symbols are experimental measurements: $\bullet$ at $y=0 \mathrm{~cm}$ (inlet); $\Delta$ at $y$ $=20 \mathrm{~cm}$; at $y=40 \mathrm{~cm} ; \bigcirc$ at $y=60 \mathrm{~cm}$. 


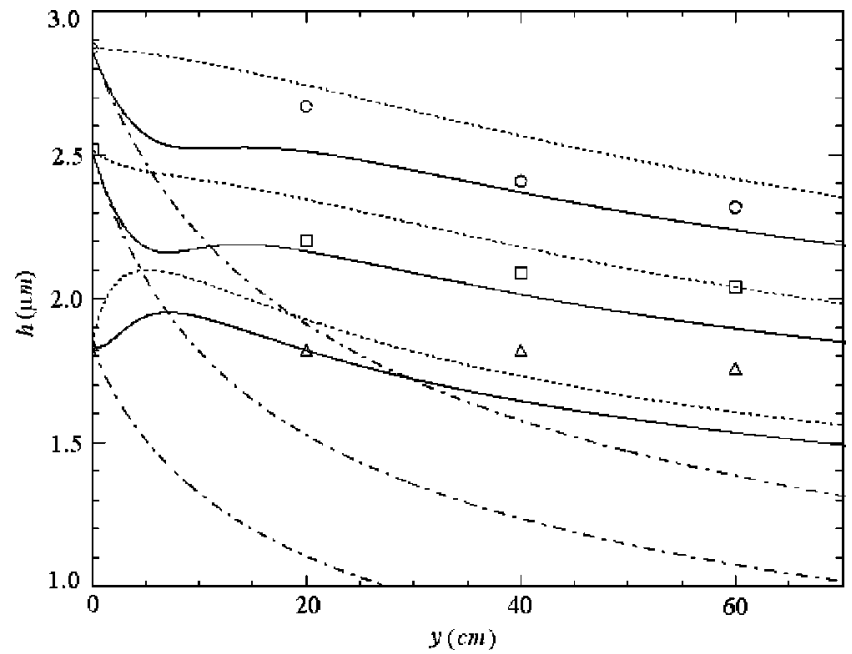

FIG. 9. The streamwise development of the computed film thickness compared with the experimental data (symbols). Solid, dotted, and dash dotted lines are results of model (a), model (b), and the no-air-drag model.

one. The velocity profiles shown by the experimental data however have larger gradients near the walls, compared to those in model (b). A proper velocity profile instead of a polynomial of degree 2 might be employed in the mathematical models to improve the agreement.

Figure 9 shows the computed variations of the film thickness together with the experimental measurements. The thinning process is much slowed down by the air drag (causing less acceleration). The measured film thickness is approximately in between the computed thickness of model (a) and that of model (b).

The variation of the film boundary layer thickness $\delta_{f}$ is shown in Fig. 10. The film boundary layer thickness drops rapidly near $y=0$. This is because when the soap solution flows downward from the divergent channel into the parallel section, the flow is actually jet-like. The momentum diffuses toward the two sides before the wall effect starts to function.

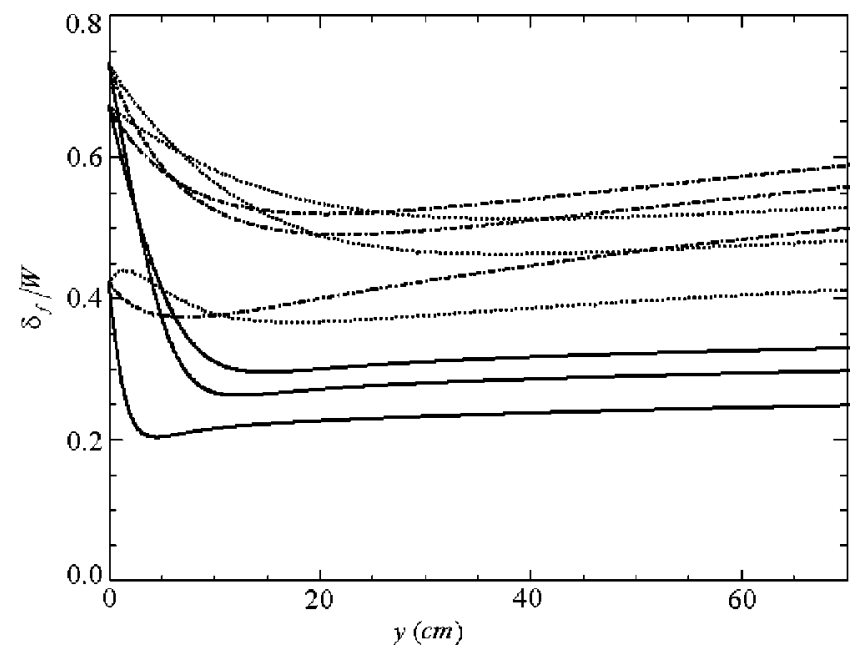

FIG. 10. The streamwise development of the computed film boundary layer thickness. Solid, dotted, and dash dotted lines are results of model (a), model (b), and the no-air-drag model. Each model has three curves corresponding to the three investigated cases.

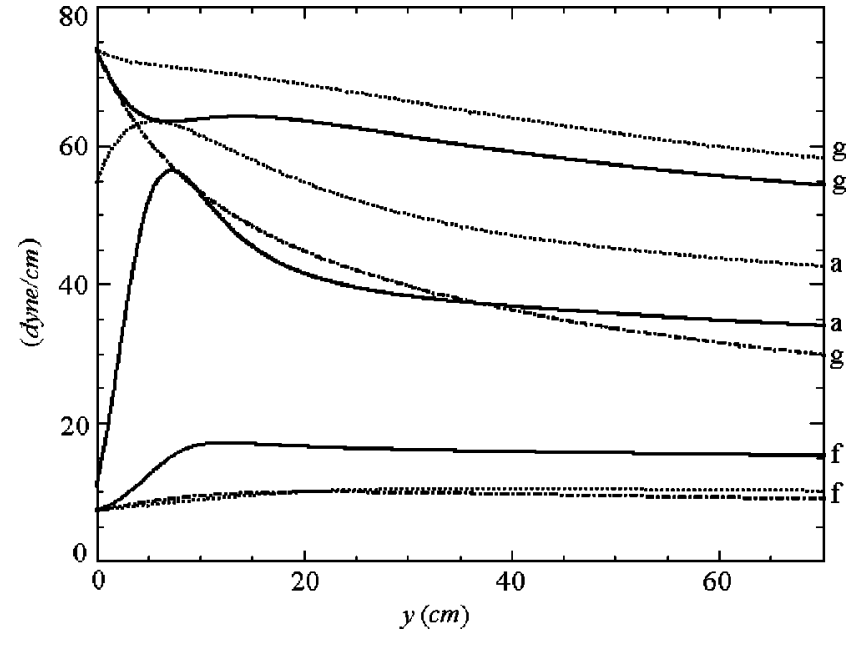

FIG. 11. The streamwise development of the gravity force $(g)$, the air drag $(a)$, and the friction force $(f)$ per unit downstream distance along the downstream distance for case 2 . Solid, dotted, and dash dotted lines are results of model (a), model (b), and the no-air-drag model.

This results in a decreasing film boundary layer thickness (measured from the wall) and consequently in an increasing area of film potential region. The latter in turn causes the total air drag force per unit downstream distance to rise occasionally, as shown by the peaks observed in the curves of the air drag forces in Figs. 11 and 12. The streamwise development of the gravity force, the air drag, and the film friction per unit downstream distance are shown in Figs. 11 and 12. The film boundary layer thickness stops decreasing at about $y=10 \mathrm{~cm}$ and from then on increases slowly. It becomes almost "saturated." A balance between the thinning due to acceleration and the thickening due to viscous diffusion is seemingly obtained. Notice that the boundary layer thickness under no air drag effect is found to be the thickest, although the flow speeds up the most (Fig. 6). A third possible mechanism to explain this is the constancy of the volume flow rate of the soap solution. As the flow speeds up rapidly, the film

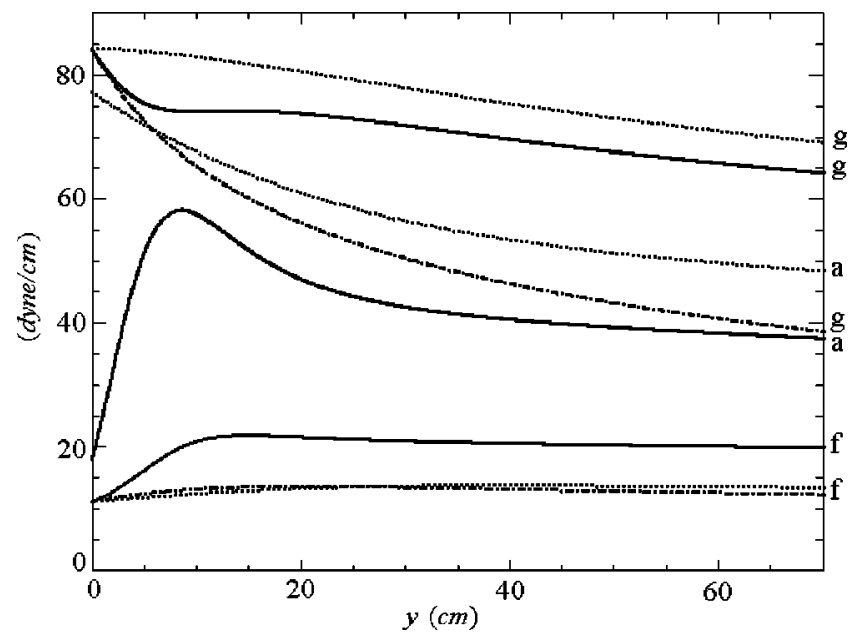

FIG. 12. The streamwise development of the gravity force $(\mathrm{g})$, the air drag $(a)$, and the friction force $(f)$ per unit downstream distance along the downstream distance for case 3. Solid, dotted, and dash dotted lines are results of model (a), model (b), and the no-air-drag model. 
boundary layer thickness can grow not only by viscous diffusion but also by the requirement of reducing the film potential region so that the volume flow rate can be maintained constant.

As seen in Figs. 11 and 12, both the gravity force and the air drag decrease with the downstream distance. This is because the film becomes thinner and the air boundary layer never stops growing. The film friction seemingly approaches a constant value, implying a balance between the slowly accelerating $V_{f}$ and a slowly growing $\delta_{f}$. Gravity nonetheless is never completely balanced by the sum of the air drag and the film friction, even at a farther downstream distance (not shown herein).

\section{CONCLUSIONS}

In this work, a Von Karman integral analysis is applied to both the film motion and the nearby, not confined, air motion in a continuously running gravity-driven soap-film tunnel. With the use of velocity profiles of polynomials of degree 2 and the Newtonian-fluid assumption, the air drag and film friction can be evaluated. The model results are compared with experimental measurements with a reasonable agreement. The analysis shows, qualitatively at least, that the film motion is found to be much slowed down by the air drag; the film boundary layer thickness decreases first, increases later on, and finally seemingly gets saturated, implying a balance between the thinning process due to acceleration and the thickening process due to viscous diffusion; the growth of the air boundary layer however never stops; and consequently, the sum of the film friction and the air drag cannot balance gravity completely.

\section{ACKNOWLEDGMENTS}

Authors are particularly grateful for valuable suggestions in designing the vertical soap-film tunnel from Professor W.
I. Goldburg (University of Pittsburgh). This work was supported by the National Science Council of the Republic of China under Grant No. NSC 87-2212-E-212-008.

${ }^{1}$ R. H. Kraichnan, "Inertial ranges in two-dimensional turbulence," Phys. Fluids 10, 1417 (1967).

${ }^{2}$ G. K. Batchelor, "Computation of the energy spectrum in homogeneous two-dimensional turbulence," Phys. Fluids 12, II-233 (1969).

${ }^{3}$ Y. Couder, "The observation of a shear flow instability in a rotating system with a soap membrane," J. Phys. (France) Lett. 42, 429 (1981).

${ }^{4}$ M. Gharib and P. Derango, "A liquid film (soap film) tunnel to study two-dimensional laminar and turbulent shear flows," Physica D 37, 406 (1989).

${ }^{5}$ H. Kellay, X. L. Wu, and W. Goldburg, "Experiments with turbulent soap films," Phys. Rev. Lett. 74, 3975 (1995).

${ }^{6}$ Y. Couder, J. M. Chomaz, and M. Rabaud, "On the hydrodynamics of soap films," Physica D 37, 384 (1989).

${ }^{7}$ Y. Couder, "Two-dimensional grid turbulence in a thin liquid film," J. Phys. (France) Lett. 45, 353 (1984).

${ }^{8}$ M. Beizaie and M. Gharib, "Fundamentals of a liquid (soap) film tunnel," Exp. Fluids 23, 130 (1997).

${ }^{9}$ X. Wu, B. K. Martin, H. Kellay, and W. Goldburg, "Hydrodynamic convection in a two-dimensional Couette cell," Phys. Rev. Lett. 75, 236 (1995).

${ }^{10}$ M. A. Rutgers, X. L. Wu, and R. Bhagavatula, "Two-dimensional velocity profiles and laminar boundary layers in flowing soap films," Phys. Fluids 8, 2847 (1996).

${ }^{11}$ B. K. Martin, X. L. Wu, W. I. Goldburg, and M. A. Rutgers, "Spectra of decaying turbulence in soap films," Phys. Rev. Lett. 80, 3964 (1998).

${ }^{12}$ M. Rivera, P. Vorobieff, and R. E. Ecke, "Turbulence in flowing soap films: Velocity, vorticity and thickness fields," Phys. Rev. Lett. 81, 1417 (1998).

${ }^{13}$ C.-Y. Wen and C.-Y Lin, "Two-dimensional vortex shedding of a circular cylinder," Phys. Fluids 13, 557 (2001).

${ }^{14}$ J. M. Chomaz, "The dynamics of a viscous soap film with soluble surfactant," J. Fluid Mech. 442, 387 (2001).

${ }^{15}$ A. Roshko, "On the development of turbulent wakes from vortex streets," NACA Report 1191, 1954.

${ }^{16}$ A. L. Rusanov and V. V. Krotov, "Gibbs elasticity of liquid films," Prog. Surf. Membr. Sci. 13, 415 (1979). 